\title{
Sonografie peripherer Nerven: Allgemeine Methodik und Untersuchungstechnik
}

\section{Sonography of Peripheral Nerves: General Methodology and Examination Technique}

\author{
Autor \\ Ulf Schminke \\ Institut \\ Klinik und Poliklinik für Neurologie, Universitätsmedizin \\ Greifswald \\ Schlüsselwörter
Sonografie, periphere Nerven, Methodik, Untersuchungs-
technik \\ Key words \\ sonography of peripheral nerves, methodology, examination \\ technique \\ Bibliografie \\ DOI https://doi.org/10.1055/a-0594-1351 \\ Klin Neurophysiol 2018; 49: 105-110 \\ (c) Georg Thieme Verlag KG Stuttgart · New York \\ ISSN 1434-0275 \\ Korrespondenzadresse \\ Prof. Ulf Schminke \\ Klinik und Poliklinik für Neurologie \\ Universitätsmedizin Greifswald \\ Ferdinand-Sauerbruch-Straße \\ 17475 Greifswald \\ ulf.schminke@uni-greifswald.de
}

\section{ZUSAMMENFASSUNG}

Die Sonografie peripherer Nerven hat sich in den vergangenen Jahren zu einem wichtigen Baustein der Diagnostik von Erkrankungen des peripheren Nervensystems entwickelt. Kenntnisse über morphologische Veränderungen peripherer Nerven und deren umgebendes Gewebe ergänzen die mittels Elektrophysiologie gewonnen Information über eine gestörte Funktion der Nerven, wodurch sich die Sicherheit einer Diagnosestellung erhöht. Der Artikel gibt eine Übersicht über die allgemeine Methodik inklusive der Anforderungen an Hardware und Software der Ultraschallgeräte. Er gewährt außerdem eine detaillierte Einführung in die Untersuchungstechnik und gibt Empfehlungen zur Dokumentation und Auswertung der Untersuchung.

\section{ABSTRACT}

In the last decade, the sonography of peripheral nerves has emerged as an important diagnostic tool for diseases of the peripheral nervous system. Information on morphologic changes of peripheral nerves and their surrounding tissue complements information on a disturbed function as shown by electrodiagnostic tests and enhances the diagnostic quality. The present article gives an overview on the general methodology including requirements for hardware and software of ultrasound devices. It further delivers a detailed introduction in the examination technique and provides recommendations for documentation and interpretation of findings.

\section{Einleitung}

Die Sonografie peripherer Nerven hat sich in den vergangenen Jahren zu einem wichtigen Baustein der Diagnostik von Erkrankungen des peripheren Nervensystems entwickelt. Kenntnisse über morphologische Veränderungen peripherer Nerven und deren umgebendes Gewebe ergänzen die mittels Elektrophysiologie gewonnen Information über eine gestörte Funktion der Nerven, wodurch sich die Sicherheit einer Diagnosestellung erhöht [1,2]. Die Qualität einer Ultraschall-Untersuchung ist allerdings in hohem Maße abhängig vom Ausbildungs- und Kenntnisstand der Untersucher, sodass ein Bedarf für eine umfassende Darstellung der Methode existiert.

Der folgende Artikel bildet den Auftakt zu einer Reihe aus insgesamt 8 Übersichtsartikeln zur Ultraschalldiagnostik bei Erkran- kungen des peripheren Nervensystems und bei neuromuskulären Erkrankungen, die zusammen genommen einen vollständigen Überblick über die Methode und deren Anwendung bei unterschiedlichen Indikationen geben sollen. Der erste Artikel hat zum Ziel, einen Überblick über die allgemeine Methodik der Sonografie peripherer Nerven und eine Einführung in die Untersuchungstechnik zu geben. Weitere Artikel dieser Reihe werden die sonografische Anatomie des peripheren Nervensystems sowie die Anwendung der Ultraschalldiagnostik bei Nervenkompressionssyndromen, neuromuskulären Erkrankungen, Nervenscheidentumoren und Ganglien, immunvermittelten Neuropathien, traumatischen Nervenläsionen und bei Sonografie-gestützten Injektionen beinhalten. 


\section{Anforderungen an die Hardware der Ultraschallgeräte}

Bereits 1988 gelang es mit der damals üblichen Gerätetechnik unter Verwendung von 5 bzw. 7,5 MHz-Schallköpfen die großen Nerven der oberen und unteren Extremitäten darzustellen [3-5]. Natürlich können diese Nerven auch heute noch mit älteren und einfacheren Ultraschallgeräten dargestellt werden. Sollen allerdings dünnere Nerven oder solche Nerven untersucht werden, die sich aufgrund ihrer topografischen Lagebeziehungen deutlich schwieriger vom umliegenden Gewebe abgrenzen lassen, stoßen ältere Ultraschallgeräte, wie sie in den Publikationen vor der Jahrtausendwende verwendet wurden, schnell an ihre Grenzen. Insbesondere für die Evaluation der faszikulären Binnenstrukturen der Nerven und deren pathologische Veränderungen sind hochauflösende Schallköpfe und eine verbesserte Geräte-Software unabdingbar. Hierbei sind insbesondere 2 Faktoren von essenzieller Bedeutung: 1. eine hohe Auflösung und 2. ein guter Kontrast gegenüber dem umliegenden Gewebe.

Eine verbesserte Auflösung kann durch die Verwendung einer höheren Sendefrequenz des Ultraschalls bewirkt werden, wobei allerdings eine höhere Sendefrequenz zulasten einer geringeren Eindringtiefe geht. Somit sind für oberflächennahe Nerven BreitbandSchallköpfe mit oberen Sendefrequenzen von 16-18 MHz zu empfehlen, mit denen eine axiale Resolution, d. h. eine Auflösung in der Ausbreitungsrichtung des Ultraschallstrahls von 150-250 $\mu \mathrm{m}$ erreicht werden können. Dagegen sind für tiefer gelegene Nerven wie bspw. für den N. ischiadicus obere Sendefrequenzen von 8-10 MHz besser geeignet. Die laterale und elevationale Resolution, d. h. die Auflösung senkrecht zur Richtung des Ultraschallstrahls und die Schichtdicke werden durch den Grad der Fokussierung des
Ultraschallstrahls bestimmt, der wiederum abhängig ist von der technischen Beschaffenheit der Schallköpfe. Möglicherweise kann zukünftig durch die Entwicklung von Matrixsonden die Auflösung noch weiter verbessern werden. Um während der Untersuchung eine optimale Auflösung zu erreichen, ist es daher unbedingt erforderlich, die Fokuszone des Ultraschallstrahls stets an die Tiefe der zu untersuchenden Struktur anzupassen. > Abb. 1 verdeutlicht den Einfluss der Fokussierung auf die Schärfe des Ultraschallbildes. Moderne hochauflösende Schallköpfe müssen dabei nicht exklusiv an große sog. High-end-Ultraschallgeräte gebunden sein. Bei den meisten Herstellern können inzwischen hochauflösende Schallköpfe auch an moderne portable Ultraschallgeräten angeschlossen werden, ohne dass dies mit hohen Einbußen an Bildqualität verbunden ist.

Grundsätzlich sollte die Untersuchung des Nervensystems mit einem Linear-Array-Schallkopf durchgeführt werden, um eine Anatomie-getreue Abbildung der zu untersuchenden Strukturen zu gewährleisten. Die Sendefrequenz sollte für die Untersuchung der meisten Fragestellungen mindestens $10 \mathrm{MHz}$ betragen. Für Fragestellungen, bei denen in erster Linie die Zu- oder Abnahme des Durchmessers bzw. der Querschnittsfläche des Nervs von Interesse ist, sind Sendefrequenzen von 10-13 MHz durchaus ausreichend. Dies ist der Fall bei Nervenkompressionssyndromen wie dem Karpaltunnel- oder Kubitaltunnel-Syndrom, bei der Darstellung von Neuromen infolge traumatischer Nervenläsionen oder bei der Darstellung von Nervenscheidentumoren und Ganglien peripher Nerven. Bei Fragestellungen, bei denen die Beurteilung der Binnenstrukturen eines Nervs bzw. der morphologischen Veränderungen einzelner Faszikel von Interesse sind, sind höhere Sendefrequenzen von 17-18 MHz zu empfehlen. Die Beurteilung der Textur im Inneren des Nervs ist von besonderer Bedeutung bei traumatischen Lä-

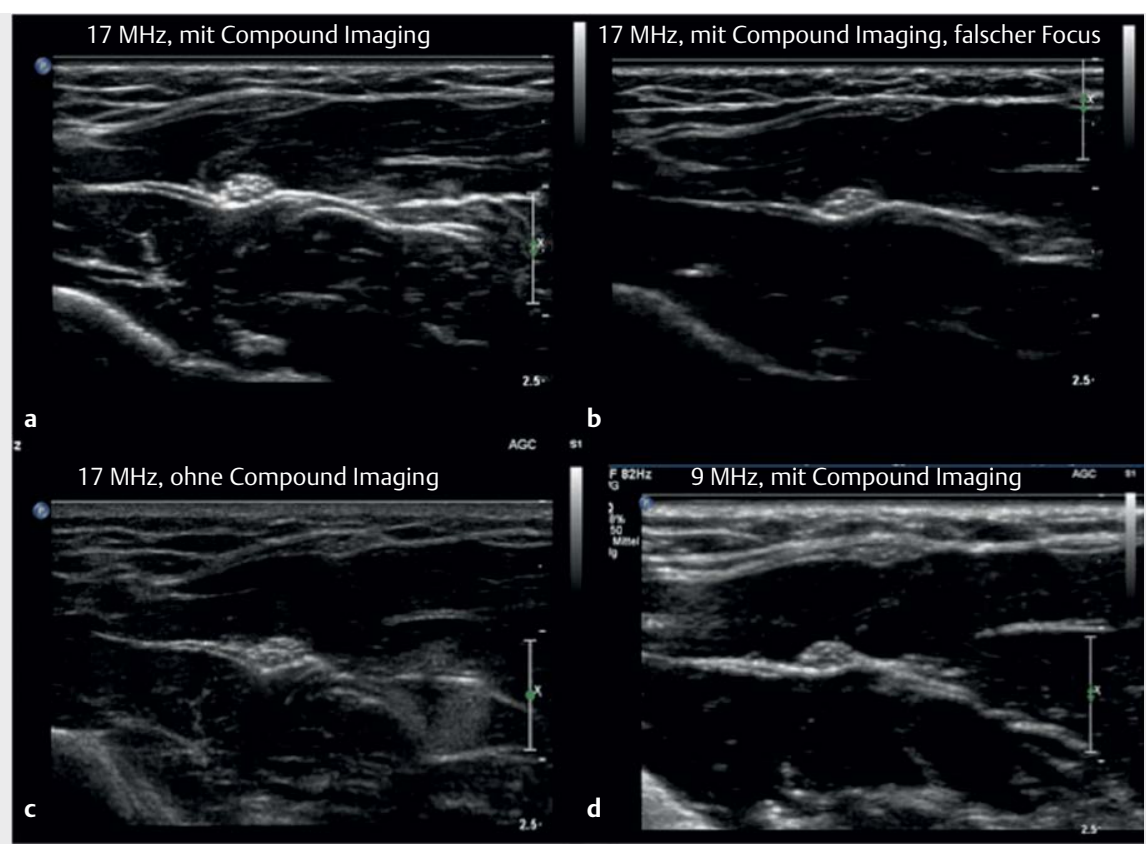

- Abb. 1 Darstellung des N. medianus bei unterschiedlichen Einstellungen des Ultraschallgerätes: a optimale Einstellung mit einer Sendefrequenz von $17 \mathrm{MHz}$, Compound Imaging eingeschaltet und adäquate Fokusposition; b gleiche Einstellung, aber Fokusposition zu weit oberflächlich; c Sendefrequenz $17 \mathrm{MHz}$, adäquate Fokusposition, aber ohne Compound Imaging; d Sendefrequenz $9 \mathrm{MHz}$, Compound Imaging eingeschaltet und adäquate Fokusposition. 
sionen mit intaktem Epineurium, bei inflammatorischen Neuropathien, aber auch bei Kompressionen, bei denen Verdickungen des Epineuriums und ödematöse Veränderungen einzelner Faszikel im Vordergrund stehen. Hohe Sendefrequenzen sind außerdem erforderlich für die Darstellung kleinerer oberflächennaher Nerven wie der R. superficialis $n$. radialis, der N. suralis oder der N. peronaeus superficialis.

\section{Anforderungen an die Geräte-Software}

Ein guter Kontrast und somit eine gute Abgrenzung peripherer Nerven vom umliegenden Gewebe ist essentiell für die Untersuchung von Nerven, die aufgrund ihrer topografischen Lage nicht einfach im Ultraschallbild zu erkennen sind. Diese wird erleichtert durch verschiedene Software-Algorithmen zur Reduzierung von Strukturartefakten, sog. Speckles.

\section{Compound Imaging}

Compound Imaging gehört zur Basis-Ausstattung der meisten modernen Ultraschallgeräte, wird aber je nach Hersteller mit unterschiedlichen Namen versehen. Durch Reflexion und Streuung des Ultraschalls an multiplen Reflektoren in homogenem Gewebe kommt zur Überlagerung von Ultraschallwellen, wodurch ein charakteristisches Interferenzmuster entsteht. Durch eine Softwaregesteuerte Auslenkung des Ultraschallstrahls werden diese Reflektoren aus unterschiedlichen Winkeln beschallt, sodass die reflektierten Ultraschallwellen zu unterschiedlichen Interferenzbildern führen. Durch Mittelung dieser Bilder kommt es zu einer Glättung des Ultraschallbildes, sodass Gewebe homogener erscheinen und Oberflächen schärfer zur Darstellung kommen. Dies gilt insbesondere für runde oder ovale Oberflächen wie dem Epineurium der Nerven im Querschnittsbild.

Neben dem Compound Imaging verfügen die meisten Ultraschallgeräte über weitere herstellerspezifische Software-Algorithmen, die Muster von benachbarten Pixeln analysieren und über eine schärfe Darstellung von Oberflächenkonturen zu einer Verbesserung der Bildqualität führen.

\section{Harmonic Imaging}

Bei der Transmission von Ultraschall durch organisches Gewebe ist in Abhängigkeit von der zurückgelegten Strecke die Ausbreitungsgeschwindigkeit des Schalls nicht uniform, wodurch sich unter anderem auch harmonische Schwingen ausbilden, die aus einem Vielfachen der ursprünglich gesendeten Fundamentalfrequenzen bestehen. Beim Harmonic Imaging werden lediglich die harmonischen Anteile des Frequenzspektrums des reflektierten Ultraschalls zur Generierung des Ultraschallbildes verwendet. Da die harmonischen Frequenzanteile erst im Gewebe entstehen, müssen sie nur einmal die Strecke bis zum Schallkopf zurücklegen und werden daher auf ihrem Weg durch das Gewebe weniger deformiert als die Fundamentalfrequenzen, die die Strecke 2-mal zurücklegen müssen. Dies führt bei größeren Untersuchungstiefen zu schärferen Bildern, bringt aber bei der Untersuchung von oberflächennah gelegenen Nerven keinen wesentlichen Vorteil, so dass auf Harmonic Imaging in der Regel verzichtet werden kann.

\section{Untersuchungstechnik}

Da die Sonografie prinzipiell die elektrophysiologische Untersuchung einer gestörten Funktion der Nerven ergänzen soll, sollten im Idealfall beide Untersuchungen im gleichen Raum oder zumindest in räumlicher Nähe stattfinden. Das ist häufig noch nicht realisierbar. Zumindest aber sollte der Untersucher die zuvor erhobenen klinischen und elektrophysiologischen Befunde gut kennen, um gezielt die Ultraschalluntersuchung an die individuelle Fragestellung anpassen zu können. Besser ist natürlich, wenn beide Untersuchungen vom gleichen Untersucher durchgeführt werden. Für die Ultraschalluntersuchung sollte der Raum abgedunkelt werden. Je nach Fragestellung kann die Untersuchung im Sitzen oder im Liegen erfolgen. Wichtig ist jedoch, dass der Patient eine entspannte Position einnehmen kann. Eine im Raum freistehende Untersuchungsliege bzw. ein freistehender Untersuchungsstuhl und ein auf Rollen beweglicher Stuhl für den Untersucher erleichtern den Zugang zu der jeweils zu untersuchenden Körperregion.

\section{Einstellungen des Sonografie-Gerätes}

Die Sonografie peripher Nerven erfolgt im B- (brightness-) Bild Modus. Bei den meisten Ultraschallgeräten existieren vordefinierte Pre-Sets, bei denen die technischen Einstellungen an die jeweils zu untersuchenden Organsystemen angepasst wurden. Falls keine Pre-Sets für die Untersuchung peripherer Neven vorgegeben sind, eignet sich am besten das ,Small Parts'-Pre-Set für die Untersuchung peripherer Nerven. Um eine optimale Auflösung bzw. ein möglichst scharfes Bild zu erhalten, ist es wichtig, die Fokus-Position auf die Tiefe der zu untersuchenden Struktur einzustellen und auch während der Untersuchung die Fokus-Position stets der Lage des Nervs anzupassen. Die B-Bild-Verstärkung, also die „Helligkeit“ des Bildes sollte über den Tiefenausgleich (bei den meisten Geräten über ein Set von Schieberegler) so eingestellt werden, dass sowohl an der Oberfläche als auch in der Tiefe des Bildes eine gleichmäßige „Helligkeit“ besteht. Da die Dämpfung des Ultraschalls je nach Körperregion unterschiedlich ist, muss auch während der Untersuchung die B-Bild-Verstärkung (bei den meisten Geräten über einen Drehknopf) stets optimiert werden. Wenn eine Unterscheidung zwischen Nerv und Blutgefäß im B-Bild Modus nicht zweifelsfrei gelingt, kann über die Duplexsonografie die Strömung im Blutgefäß farbig dargestellt werden, wobei entweder die Puls-Repetitions-Frequenz (PRF) an die zu erwarteten niedrigen Strömungsgeschwindigkeiten in den zumeist venösen Blutgefäßen der Extremitäten angepasst oder der Power-Doppler zur Farbcodierung gewählt werden muss. Eine Zusammenfassung der technischen Empfehlungen ist in > Tab. 1 aufgelistet.

\section{Untersuchungsgang}

Zum Auffinden der Nerven ist es deutlicher einfacher, die Untersuchung im Querschnittsbild zu beginnen, da sich im Querschnittsbild die Nerven in ihrer charakteristischen faszikulären Struktur darstellen, die einer Honigwabe oder einem Blumenkohl ähnelt ( $\triangleright$ Abb. 2). Dabei ist auf einen streng senkrechten Beschallungswinkel zu achten. Die einzelnen Faszikel kommen im Ultraschallbild echoarm zur Darstellung und sind umgeben von echoreichem Perineurium. Die Gesamtheit der Faszikel und das Perineurium wiederum sind umgeben vom ebenfalls echoreichen Epineurium. Streng genommen hat ein Nerv in der Regel mehr Faszikel als im Ultraschall 
- Tab. 1 Technische Empfehlungen.

\begin{tabular}{|l|l|} 
Schallkopf & Breitband Linear-Array Schallkopf \\
\hline Sendefrequenz des Schallkopfes & $\geq 10 \mathrm{MHz}$, besser: $17-18 \mathrm{MHz}$ \\
\hline Pre-Set & „small parts“ oder Nerven-Pre-Sets \\
\hline Compound Imaging & ja \\
\hline Second Harmonic Imaging & nein \\
\hline Untersuchungstiefe & an Lokalisation des Nervs anpassen \\
\hline Fokusposition & an Lokalisation des Nervs anpassen \\
\hline tiefenabhängige Verstärkung & optimieren \\
\hline B-Bild Verstärkung (Helligkeit) & optimieren \\
\hline
\end{tabular}

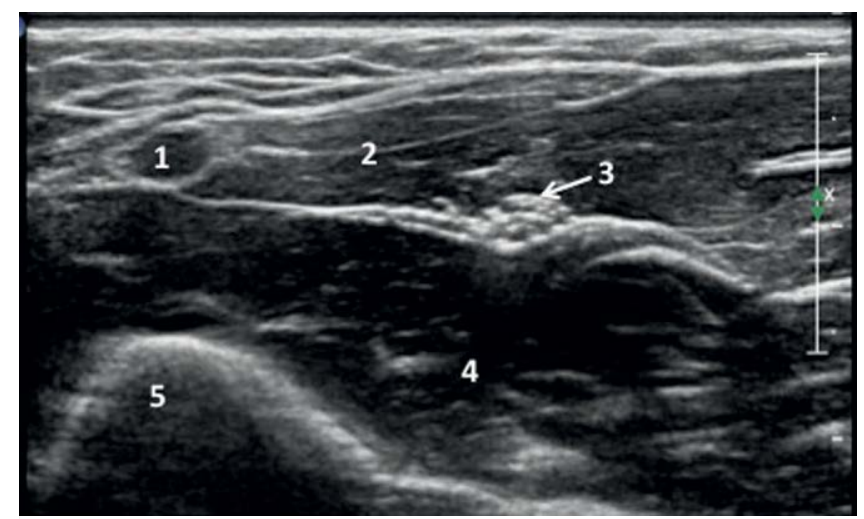

Abb. 2 Darstellung des N. medianus im distalen Drittel des Unterarms zwischen den oberflächennahen und tiefen Beugemuskeln. Das Querschnittsbild zeigt die typische wabenförmige Echotextur des Nervs mit echoarmen Faszikeln, die von echoreichem Perineurium umgeben sind, während der gesamte Nerv vom ebenfalls echoreichen Epineurium umgeben ist. 1: A. radialis; 2: oberflächennahe Flexoren; 3: N. medianus; 4: tiefe Flexoren; 5: Radius.

dargestellt werden können, da Faszikel, die durch ein dünnes Perineurium getrennt sind, als ein einzelner Faszikel oder besser gesagt als ein einzelnes Faszikelbündel erscheinen [6]. Durch Drehung des Schallkopfes um $90^{\circ}$ wird der Nerv im Längsschnitt abgebildet, in der er eine kabelförmige, tubuläre Struktur aufweist ( $>$ Abb. $\mathbf{3}$ ). Am Hand- und Sprunggelenk kann es mitunter schwierig sein, den Nerv von benachbarten Sehnen zu unterscheiden. Hier kann die höhere Anisotropie der Sehnen im Vergleich zum Nerv zur Differenzierung genutzt werden. Gewebe mit hoher Anisotropie reflektieren den Ultraschall sehr stark und verhalten sich dabei wie ein Spiegel. Der Einfallswinkel des Ultraschallstrahls entspricht dem Ausfallswinkel, sodass nur bei senkrechter Beschallung der Ultraschall zum Schallkopf zurück reflektiert wird. Daraus folgt, dass Sehnen ihre Echogenität bei nicht exakt senkrechtem Beschallungswinkel deutlich stärker ändern als Nerven. Bei leichtem Kippen des Schallkopfes ändert sich die Echogenität der Sehnen erheblich, während sich die Echogenität des Nervs nur wenig ändert ( $\triangleright$ Video 1).

Mögliche Fehlerquellen bei der Untersuchung sind in $>$ Tab. 2 aufgeführt.

Bei der Untersuchung ist es im allgemeinen üblich, den Schallkopf so auszurichten, dass die Markierung am Schallkopf, die den linken Rand des Ultraschallbildes anzeigt, vom Körperzentrum weg zeigt. Zum Beispiel befinden sich bei der Untersuchung des Unter-
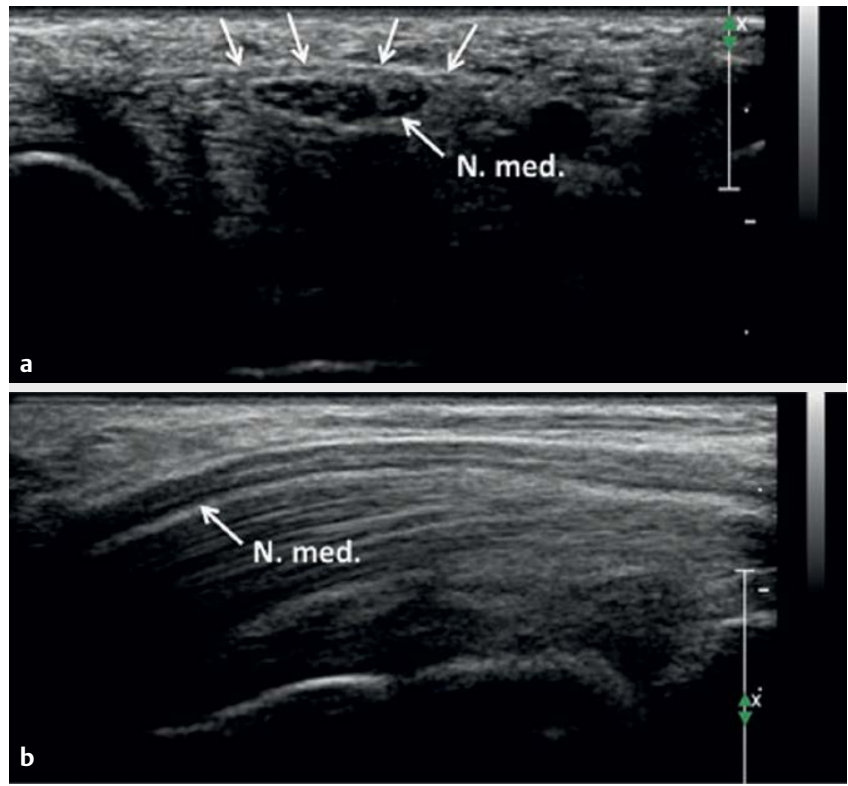

- Abb. 3 Darstellung des N. medianus am Handgelenk. a transversale Schnittebene: Der Nerv befindet sich unter dem echoarmen Retinaculum flexorum (Pfeile); b longitudinale Schnittebene.

\section{VIDEO 1

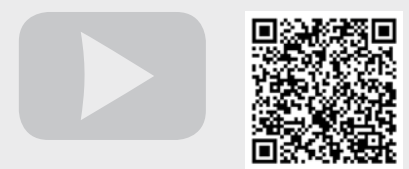 \\ https://doi.org/ \\ 10-1055-a-0594-1351 \\ - Video 1 Darstellung des N. medianus und der Sehnen der Finger- beugemuskeln unterhalb des Retinaculum flexorum am Handgelenk. Durch leichtes Kippen des Schallkopfes ändert sich die Echogenität der Sehnen erheblich, während sich die Echogenität des Nervs nur wenig ändert.}

arms die radiale Seite links und die ulnare Seite rechts im Ultraschallbild. Dies hat den Vorteil, dass die Ultraschallbilder der rechten und der linken Extremitäten in gleicher Orientierung ausgerichtet sind. Neuerdings wird in Publikationen allerdings zunehmend auch eine mit dem MRT vergleichbare Ausrichtung der Ultraschallbilder angewandt, bei der die linke Körperseite stets am rechten Bildrand zu finden ist (man schaut also von unten in den Körper). Dies führt aber dazu, dass die Ultraschallbilder der rechten und der linken Extremitäten spiegelbildlich zueinander sind.

Es empfiehlt sich die Untersuchung an definierten Landmarken zu beginnen, an denen der Nerv einfach aufzufinden ist. Am einfachsten ist der N. medianus im distalen Drittel des Unterarms aufzufinden. Er liegt dort zwischen den oberflächennahen und tiefen Beugemuskeln und ist an seiner faszikulären Struktur gut vom umgebenden Gewebe zu differenzieren ( $\triangleright$ Abb. 2). Von hier aus sollte der Nerv nach proximal und nach distal verfolgt werden.

( $\triangleright$ Video 2 und 3 )

Am Handgelenk befindet sich der N. medianus unmittelbar unterhalb des Retinaculum flexorum, das sich im Ultraschallbild als 
- Tab. 2 Mögliche technische Fehler und deren Abhilfe.

\section{Gerätefehler oder Artefakt}

senkrechte schwarze Streifen im Bild

oberflächliche Strukturen zu hell

Zielstruktur unscharf

tiefe Strukturen nicht gut darstellbar

Anisotropie-Artefakte (Verlust der Echogenität der Binnenechos eines Nervs)

Reverberations-Artefakte (Mehrfachreflexionen) helle horizontale Streifenartefakte
Abhilfe

zu wenig Ultraschallgel oder einzelne piezoelektrische Elemente des Schallkopfs defekt

Tiefenausgleich optimieren

Fokusposition optimieren

Schallkopf mit niedrigerer Frequenz verwenden

Beschallungswinkel nicht senkrecht

Mehr Ultraschall-Gel verwenden oder Beschallungswinkel ändern

\section{VIDEO 2}
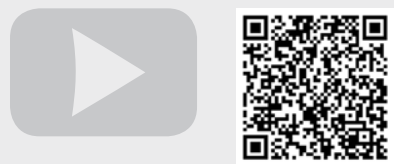

https://doi.org/

10-1055-a-0594-1351

- Video 2 Untersuchung des N. medianus im Querschnittsbild durch Verschieben der Sonde vom distalen Unterarm-Drittel nach distal bis zum Handgelenk.

\section{VIDEO 3}
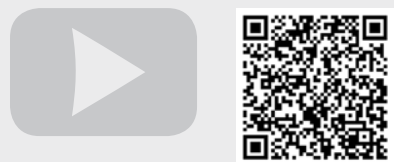

https://doi.org/

10-1055-a-0594-1351

- Video 3 Untersuchung des N. medianus im Querschnittsbild durch Verschieben der Sonde vom distalen Unterarm-drittel nach proximal bis zur Ellenbeuge.

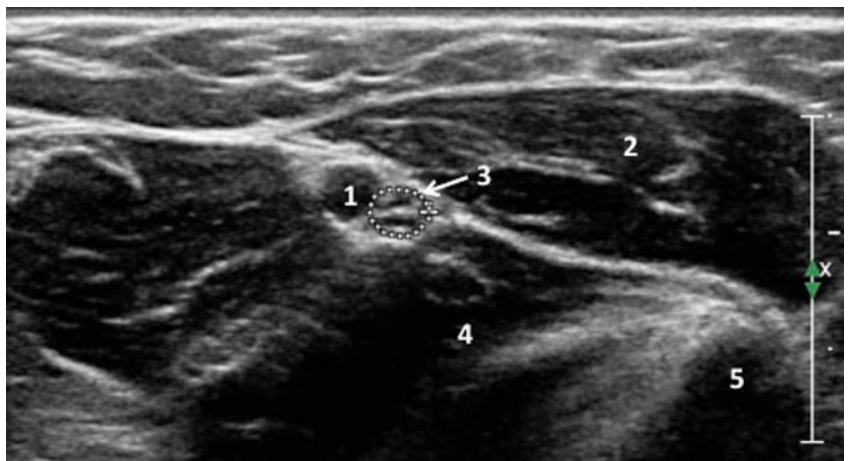

Abb. 4 Darstellung des N. ulnaris im distalen Drittel des Unterarms, der unterhalb des $\mathrm{M}$. flexor carpi ulnaris neben der $\mathrm{A}$. ulnaris verläuft. 1: A. ulnaris; 2: M. flexor carpi ulnaris; 3: N. ulnaris; 4: tiefe Flexoren; 5: Ulna. echoarmes Band darstellt ( $\mathbf{A}$ Abb. 3). Pathologische Veränderungen sollten stets sowohl im Querschnitts- als auch im Längsschnittbild untersucht werden. Auch der N. ulnaris ist am einfachsten im distalen Drittel des Unterarmes zu finden. Er verläuft ulnar der A. ulnaris unterhalb des M. flexor carpi ulnaris ( $\mathbf{A b b} \mathbf{4} \mathbf{4})$.

\section{Fallstricke und Fehlerquellen}

Bei einem nicht senkrechten Beschallungswinkel kommen Nerven nur unscharf zur Darstellung und die faszikuläre Binnenstruktur wird verwischt. Zudem wird bei einem tangential angeschnittenen Nerv die Messung der Nervenquerschnittsfläche fälschlich zu hohe Werte ergeben. Bei einer Messung des Diameters im Längsschnittbild muss ein ovaler Querschnitt des Nervs bedacht werden. Die Ausdehnung eines Nervs kann in die Breite zunehmen, ohne dass sich die bei der Diameterbestimmung gemessene Höhe ändert. Eine weitere Fehlerquelle ist die Überbewertung von morphologischen Anomalien bei elektrophysiologisch und/oder klinisch ungestörter Funktion eines Nervs.

\section{Dokumentation und Auswertung der Untersuchung}

Zur Dokumentation der Befunde sollten die elektronisch gespeicherten Bilder auf ein Dauerspeichermedium (Klinik-PACS oder externer Festplattenspeicher) transferiert werden. Alle relevanten Befunde sollten sowohl im Querschnittsbild ggf. mit Messung einer Nervenquerschnittsfläche als auch im Längsschnittbild dokumentiert werden. In Einzelfällen kann es sinnvoll sein, eine Folge transversaler Schnittebenen als kurze Videosequenz (z. B. als cine loop) zu speichern, während der Schallkopf von proximal nach distal bewegt wird. Abspeichern von cine loops sind insbesondere bei dynamischen Untersuchung vorteilhaft, wenn während einer Bewegung der Extremität in ihren Gelenken die Änderung der Lagebeziehung von Nerven zum umgebenden Gewebe, z. B. bei einer Kompressionen eines Nervs durch akzessorische Muskelbäuche untersucht werden sollen. Spielt die Vaskularisierung von Prozessen eine Rolle wie z. B. bei Nervenscheidentumoren sollte zusätzliche Bilder im Farbmodus gespeichert werden.

Die Auswertung der Untersuchungsbefunde erfolgt unter Berücksichtigung der Fragestellung. Bei fokalen Neuropathien, die mit einer umschriebenen „Verdickung“ der Nerven einhergehen, 
- Tab. 3 Normwerte von häufig untersuchten Nervensegmenten.

\begin{tabular}{|l|l|l|l|}
\hline & Böhm J. et al., 2014 [7] & Tagliafico A. et al., 2013 [8] & Busemann A., 2014 [9] \\
\hline Nervensegment & mean \pm SD & mean \pm SD & mean \pm SD \\
\hline N. medianus am Oberarm & $8,9 \pm 1,8 \mathrm{~mm}^{2}$ & $7,5 \pm 1,5 \mathrm{~mm}^{2}$ \\
\hline N. medianus am Unterarm & $5,7 \pm 1,3 \mathrm{~mm}^{2}$ & $5,5 \pm 2,2 \mathrm{~mm}^{2}$ & $6,7 \pm 1,9 \mathrm{~mm}^{2}$ \\
\hline N. medianus am Handgelenk & $8,5 \pm 1,8 \mathrm{~mm}^{2}$ & $8,2 \pm 2,3 \mathrm{~mm}^{2}$ & $5,6 \pm 1,6 \mathrm{~mm}^{2}$ \\
\hline N. ulnaris am Oberarm & $6,3 \pm 1,7 \mathrm{~mm}^{2}$ & \\
\hline N. ulnaris am Epicondylus medialis & $7,6 \pm 2,1 \mathrm{~mm}^{2}$ & $5,9 \pm 3,0 \mathrm{~mm}^{2}$ & $4,3 \pm 1,4 \mathrm{~mm}^{2}$ \\
\hline N. ulnaris am Unterarm & $5,2 \pm 1,3 \mathrm{~mm}^{2}$ & \\
\hline N. peronaeus am Fibulaköpfchen & $8,9 \pm 2,0 \mathrm{~mm}^{2}$ & & $9,5 \pm 2,8 \mathrm{~mm}^{2}$ \\
\hline N. tibialis am Malleolus medialis & $9,6 \pm 2,2 \mathrm{~mm}^{2}$ & \\
\hline mean= arithmetischer Mittelwert, SD $=$ Standardabweichung & & \\
\hline
\end{tabular}

sollte eine Messung der maximalen Nervenquerschnittsfläche erfolgen. Diese Messung erfolgt im Querschnittsbild durch Umfahren des Nervs an der Grenze zwischen echoarmen Faszikeln und dem echoreichen Epineuriums mit dem Curser der Messfunktion des Ultraschallgerätes. D. h., es wird der Umfang des Nervs innerhalb des Epineuriums gemessen. Teilweise können aber auch nur einzelne Faszikel bzw. Faszikelbündel verdickt sein. Eine ausführliche Übersicht über Normwerte von Nervenquerschnittsflächen von insgesamt 14 Nervensegmenten bei Gesunden ist bei Böhm J. et al., Ultraschall Med. 2014; 35: 459-467 publiziert [7]. Darüber hinaus werden bei Tagliafico A et al., J Ultrasound Med. 2013; 32: 457-62 weitere Normwerte von weniger häufig untersuchten Nervensegmenten der oberen Extremitäten bei Gesunden beschrieben [8]. Eine Übersicht von Normwerten häufig untersuchter Nervensegmente zeigt $>$ Tab. $\mathbf{3}$.

Bei Nervenkompressionssyndromen besteht eine Zunahme der Nervenquerschnittsflächen proximal und teils auch distal der Kompressionsstelle, während der Nerv am Ort der Kompression abgeplattet erscheint. Bei grenzwertigen Befunden ist die Bildung von Quotienten zwischen der Querschnittsfläche des Nervs an der Stelle der maximalen Verdickung im Verhältnis zu der Querschnittsfläche eines gesunden Segments sinnvoll. Bei traumatischen Nervenläsionen bildet sich bei einer Unterbrechung der Kontinuität des Nervs am proximalen Stumpf ein Neurom aus. Bei intaktem Epineurium bspw. bei Traktionsschädigungen kann es zu Veränderungen der Binnenstrukturen der Nerven (intraneurale Neurome) oder zu einem Verlust der faszikulären Struktur des Nervs im Längsschnittbild kommen. Nerven können durch Fremdkörper, Knochenfragmente oder Osteosynthesematerial komprimiert oder von Narbengewebe ummauert sein. Schwannome sind exzentrisch gelegene, runde oder ovale, meist fusiform konfigurierte, glatt begrenzte Raumforderungen mit einer inhomogenen Binnenstruktur, einer überwiegend echoarmen Echogenität, die aus der Kontinuität eines ansonsten normal aussehenden Nervs hervorgehen und gut vaskularisiert sind. Dagegen sind Neurofibrome nicht verkapselt und in der Kontinuität eines Nervs gelegen, wobei sie betroffene Faszikel spindelförmig auftreiben. Hereditäre oder immunvermittelte Neuropathien führen entweder zu einer fokalen, multifokalen oder generalisierten Zunahme der Nervenquerschnittsfläche außerhalb von Prädilektionsstellen einer Nervenkompression oder zu einer Zunahme des Kalibers einzelner Faszikel.

Eine zusammenfassende Bewertung und Einordnung der erhobenen Befunde sollte stets im Kontext mit dem klinischen und/oder elektrophysiologischen Untersuchungsbefund erfolgen. Der unkritische Einsatz bildgebender Diagnostik als alleinige Untersuchungsmodalität birgt andernfalls die Gefahr klinisch nicht relevante Zufallsbefunde fehl zu deuten.

\section{Interessenkonflikt}

Der Autor gibt an, dass kein Interessenkonflikt besteht

\section{Literatur}

[1] Schelle T. Methodik und was kann die Nervensonografie. Klin Neurophysiol 2015; 46: 79-89

[2] Schminke U. Sonographie peripherer Nerven: Relevante Indikationen für die klinische Praxis. Klin Neurophysiol 2016; 47: 173-179

[3] Fornage BD. Peripheral nerves of the extremities: Imaging with US. Radiology 1988; 167: 179-182

[4] Graif M, Seton A, Nerubai J et al. Sciatic nerve: Sonographic evaluation and anatomic-pathologic considerations. Radiology 1991; 181: 405-408

[5] Buchberger W, Schön G, Strasser K et al. High-resolution ultrasonography of the carpal tunnel. J Ultrasound Med 1991; 10: 531-537

[6] Silvestri E, Martinoli C, Derchi LE et al. Echotexture of peripheral nerves: correlation between US and histologic findings and criteria to differentiate tendons. Radiology 1995; 197: 291-296

[7] Boehm J, Scheidl E, Bereczki D et al. High-resolution ultrasonography of peripheral nerves: measurements on 14 nerve segments in 56 healthy subjects and reliability assessments. Ultraschall Med 2014; 35: 459-467

[8] Tagliafico A, Martinoli C. Reliability of side-to-side sonographic cross-sectional area measurements of upper extremity nerves in healthy volunteers. J Ultrasound Med 2013; 32: 457-462

[9] Busemann A. Sonographie peripherer Nerven bei Polyneuropathien. Dissertation. Greifswald 2013 